\title{
MACE: A Multi-Attribute Combinatorial Exchange
}

\author{
Björn Schnizler \\ schnizlereism.uni-karlsruhe.de \\ Institute of Information Systems and Management (IISM) \\ Universität Karlsruhe (TH)
}

The increasing interconnection between computers has created a vision for Grids. Within these Grids, computing resources such as processing power, storage space or applications are accessible to any participant. This accessibility has major ramifications for organizations since they can reduce costs by outsourcing nonessential elements of their IT infrastructure to various forms of service providers. Such emerging e-Utilities - providers offering on-demand access to computing resources - enable organizations to perform computational jobs spontaneously through other resources in the Grid that are not under the control of the (temporary) user (Foster et al., 2002).

With the implementation of Grid middleware, institutional arrangements are becoming increasingly important. In essence, current Grid middleware provides insufficient incentive to participate in the Grid. In this context, the application of markets for the Grid is considered to work well. By assigning a value (also called utility) to their service requests, users can reveal their relative urgency or costs to the service, which is subject to service usage constraints. If the market mechanism is properly defined, users may be provided incentives to express their true values for service requests and offers. This in turn marks the prerequisite for attaining an efficient allocation of services, which maximizes the sum of aggregate valuations (Schnizler et al., 2005).

MACE - a multi-attribute combinatorial exchange - is a suitable auction mechanism for allocating and scheduling resources in the Grid (Schnizler et al., 2006). In contrast to other approaches, the proposed mechanism accounts for the variety of services by incorporating 
time and quality as well as coupling constraints. The mechanism provides buyers and sellers with a rich bidding language, allowing for the formulation of bundles expressing either substitutabilities or complementarities. The winner determination problem maximizes the social welfare of this combinatorial allocation problem. The winner determination scheme alone, however, is insufficient to guarantee an efficient allocation of the services. The pricing scheme must be constructed in a way that motivates buyers and sellers to reveal their true valuations and reservation prices. This is problematic in the case of combinatorial exchanges, since the only efficient pricing schedule, the VCG mechanism, is not budget-balanced and must be subsidized from outside the mechanism.

The auction implements on a new pricing family for a combinatorial exchange, namely the $k$-pricing rule. In essence, the $k$-pricing rule determines the price such that the resulting surpluses to the buyers and sellers divide the entire surplus being accrued by the trade according to the ration $k$. The $k$-pricing rule is budget-balanced but cannot retain the efficiency property of the VCG payments. As a simulation illustrates, the $k$-pricing is approximative incentive compatible and, thus, a practical alternative to the VCG mechanism and highly relevant for an application in the Grid (Schnizler et al., 2006).

Future research of MACE needs to consider alternative heuristics that simplify the winner determination problem. Nature-inspired algorithms such as genetic algorithms may be adequate for an application in the service market. A further step towards a functioning Open Grid Market would be to confront the mechanism with real data in a pilot run.

\section{References}

Foster, I., Kesselman, C., Nick, J., Tuecke, S., 2002. Grid services for distributed system integration. IEEE Computer 35 (6), 37-46.

Schnizler, B., Neumann, D., Veit, D., Weinhardt, C., 2005. A multiattribute combinatorial exchange for trading Grid resources. In: Tan, Y. (Ed.), Proceedings of the 12th Research Symposium on Emerging Electronic Markets (RSEEM), Amsterdam, Netherlands. pp. 220-240.

Schnizler, B., Neumann, D., Veit, D., Weinhardt, C., 2006. Trading Grid services - a multiattribute combinatorial approach. European Journal of Operational Research, forthcoming. 